\title{
COVID-19 Adaptations for Health Service Psychology Internship Training in a Medical School
}

\author{
Heather Agazzi ${ }^{1}$ [ $\cdot$ Shadae Najmabadi ${ }^{1} \cdot$ Jacquelyn Flood $^{2} \cdot$ Danielle Cimorelli $^{1} \cdot$ Tiffany Chenneville $^{3}$
}

Accepted: 26 April 2021 / Published online: 9 May 2021

(c) The Author(s), under exclusive licence to Springer Science+Business Media, LLC, part of Springer Nature 2021

\begin{abstract}
Health service psychology internship directors confronted a myriad of training challenges in response to the COVID-19 pandemic. Specifically, internship training directors were tasked with ensuring that interns received appropriate training in each of the nine profession-wide competency (PWCs) areas while also ensuring the physical and emotional well-being of staff and trainees. The purpose of this paper is to describe one internship's approach to adapting the nine PWCs during COVID-19 in the context of an academic medical setting. Successes and challenges associated with training adaptations in the context of each of the nine PWCs will be shared along with considerations for improving academic medical training programs' ability to support interns' educational goals and training requirements throughout and following the COVID-19 pandemic.
\end{abstract}

Keywords Internship $\cdot$ Medical $\cdot$ COVID-19 $\cdot$ Adaptations $\cdot$ Profession-wide competencies

\section{Introduction}

In January 2020, few people in the United States (US) had ever heard about COVID-19 and its rapid spread across China (World Health Organization, 2020). Almost two months later, COVID-19 became part of daily news, and was central to national, state, and local discussions. Given its rapid spread and the mounting infections and associated deaths across the globe, the World Health Organization (World Health Organization, 2020) declared COVID-19 an international public health emergency. While the US government provided some guidance, each state had to create their own response to the pandemic. By late March 2020, most states in the US were implementing stay-at-home orders with only essential workers permitted to go to the workplace. Academic institutions sent college students home to finish the semester via remote learning, including graduate

Heather Agazzi

hcurtis@usf.edu

1 USF Health Department of Pediatrics, University of South Florida, 13101 N. Bruce B. Downs Blvd, Tampa, FL 33612, USA

2 Department of Psychiatry, University of South Florida, Tampa, FL, USA

3 Department of Psychology, University of South Florida, St. Petersburg, FL, USA students in the health service fields (e.g., psychology, medicine, nursing) who were pulled from clinical rotations altogether. Trainees at more advanced stages of professional clinical training programs, including medical residents and fellows, psychology interns and fellows, and their equivalents across disciplines could not simply be sent home to finish the semester, as their training requires contact hours with patients. Health service training programs were faced with having to ensure adequate clinical training opportunities while maintaining a safe training environment. Centers that had previously embraced telehealth services were ideally positioned to take on the challenge and transfer clinical care to remote platforms, while others had the additional challenge of embracing a new platform for the delivery of health care services.

In response to the COVID-19 pandemic, health service psychology internship directors were confronted with a myriad of training challenges, the measured impact of which is currently unknown. The Association of Psychology Postdoctoral and Internship Centers (APPIC) received multiple queries in late March regarding how to respond to the consequences of the COVID-19 crisis and its impact on psychology training (APPIC, 2020, March 23). Given the diversity of its member sites, APPIC encouraged sites to tailor their programs to meet the needs of patients, to support trainees' educational goals and requirements, and to keep in mind the physical and emotional well-being of staff and 
trainees. At the same time, member sites had to consider the APPIC membership criteria, the American Psychological Association's (APA) criteria, state requirements for licensure, and institution-specific directives. Our institution directives named psychologists as essential employees alongside allied health professionals and required psychologists to report to work and embrace the use of personal protective equipment (PPE) and heavy sanitation practices. Key considerations included, but were not limited to determining if interns were considered 'essential employees', maintaining a caseload of assessment and intervention cases to ensure client contact hours, safely implementing services in-person or via telehealth, accessing PPE for in-person visits, and delivering effective and appropriate supervision given the physical distancing guidelines. Health service psychology training directors had to respond swiftly to ensure their programs could adapt to the pandemic and support trainees' educational goals and requirements within the context of psychology profession-wide competencies (PWCs; Commission on Accreditation, 2017) as well as meet institutionspecific directives. In our context, institution directives were not in conflict with other association guidelines.

Arguably, psychologists are well positioned to respond to the many threats of a global pandemic with their extensive training in human communication, stress management, crisis response, and psychological interventions. Indeed, health service psychology training directors are responding to the COVID-19 pandemic through adjustments to their education of interns/fellows and clinical practice. The purpose of this paper is to describe one internship's approach to ensuring health service psychology training across the nine PWCs during COVID-19. Training adaptations in the context of each of the nine PWCs will be shared. Successes and challenges associated with these adaptations will be put forth, along with considerations for improving training programs' ability to support trainees' educational goals and training requirements throughout and following the COVID-19 pandemic. Indeed, some adaptations may prove beneficial over the longer run, such as providing more telepsychology service opportunities to increase diversity in training experiences and health psychologist preparedness for the future.

\section{Relevant Training Competencies}

The profession-wide competencies (PWCs) offer nine broad competency areas in which psychology interns must receive appropriate training by the completion of internship (American Psychological Association, 2018). These competencies are embedded within the Standards of Accreditation (SoA; Commission on Accreditation, 2017), a framework created by the Commission on Accreditation (CoA) to guide training programs in the development of their training experiences.
The PWCs provide a standard set of applied training requirements for all programs, which are measurable, specific areas of knowledge/skills/attitudes related to health service psychology and are comprised of multiple elements. The nine PWCs include: (1) research, (2) ethical/legal standards, (3) individual and cultural diversity, (4) professional values and attitudes, (5) communication and interpersonal skills, (6) assessment, (7) intervention, (8) supervision, and (9) consultation and interprofessional/interdisciplinary skills (Commission on Accreditation, 2017). The Implementation Regulation (IR) C-16 I. states that interns' competencies must be assessed in terms of multiple elements. Each program is responsible for demonstrating a sufficient number of elements articulated for each PWC so as to document adequate trainee competence, and each program must give feedback to interns at the level of elements, while reporting to the CoA at the level of the PWC. While IR C-8 I. (Commission on Accreditation, 2017) provides multiple elements for seven of the nine PWCs, programs must identify at least one program-defined element for the Research and Supervision competencies. See Table 1 for a brief description of each of the nine PWCs and associated training elements as outlined by the Commission on Accreditation (CoA) Implementing Regulations (IRs).

\section{Profession-Wide Competencies and COVID-19 Adaptations}

COVID-19 necessitated a swift transition from in-person to virtual training across PWCs. The fact that internship supervisors and interns alike were competent in remote-based technology, had personal devices, and had internet services in their home helped ease this transition. However, there were other challenges. In the sections to follow are examples of how one pediatric psychology internship program located at a large academic medical center in the southeastern U.S. adapted training elements to promote trainees' competencies in a largely virtual environment.

\section{PWC-1: Research}

In compliance with IR C-16. I., our program-defined element associated with the Research competency included coreview of manuscripts or research abstracts with the internship director, including write-up of a critical summary of strengths and weaknesses. Due to the impact of COVID-19 on professionals' daily lives, many journal editors found their usual reviewers declining the opportunity to review articles for publication. Our program took this opportunity to accept several article reviews, as this work could be conducted remotely which ensured the safety of interns and created more opportunities to meet our Research elements. 


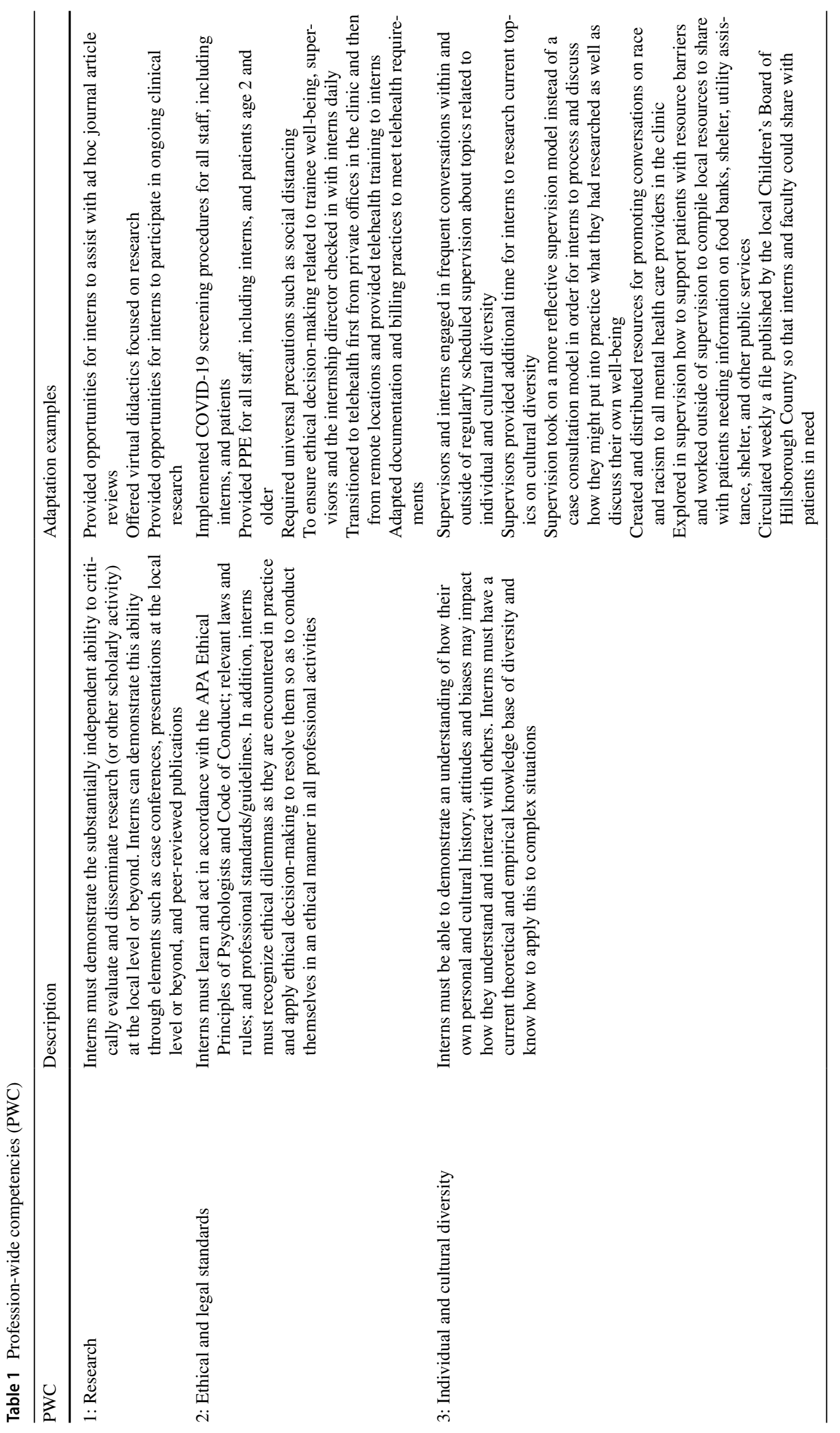




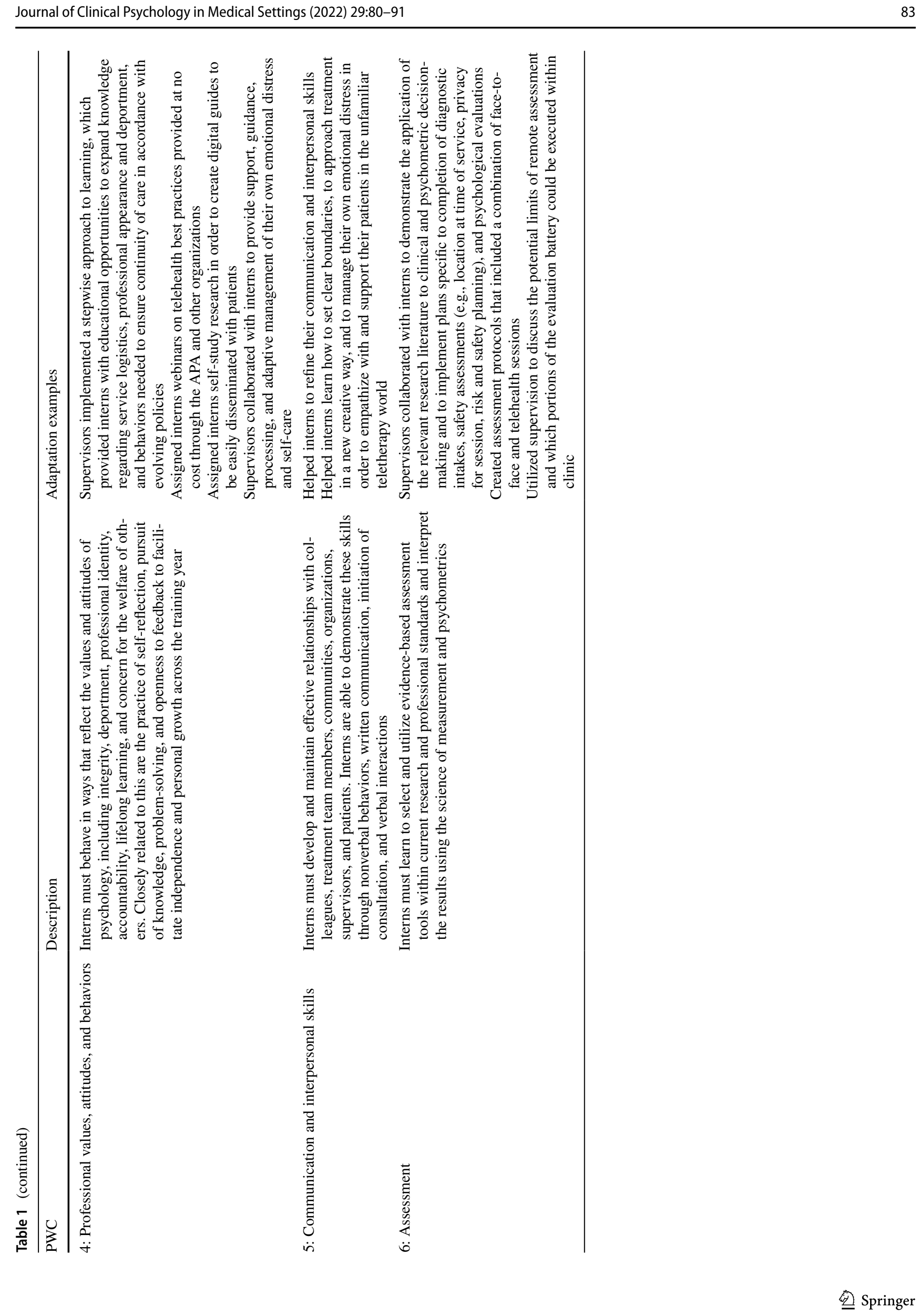




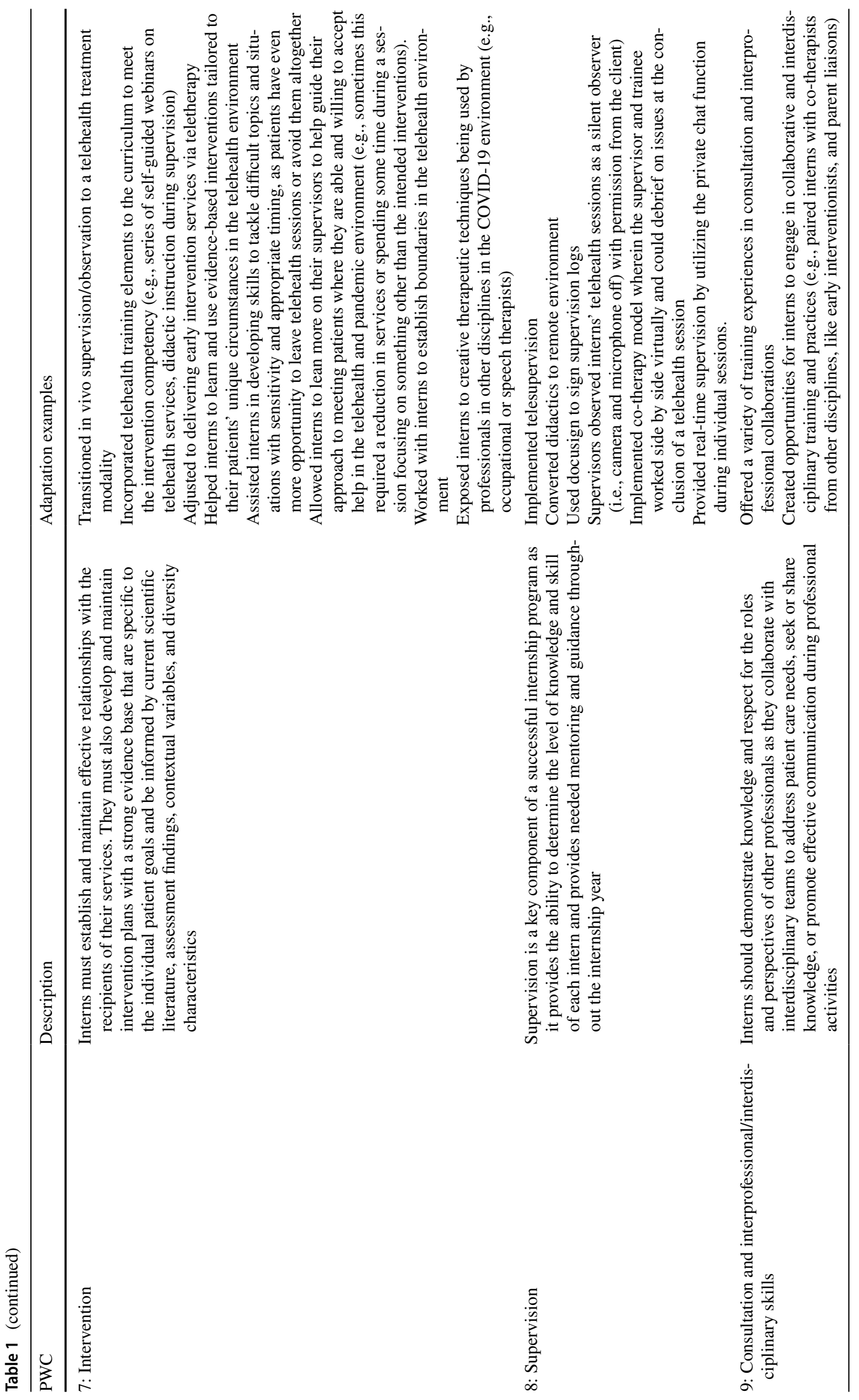


The internship director selected articles from many journals for which she is an ad hoc reviewer that were relevant to the child and adolescent training in our program including: parent training, pediatric health issues and psychosocial management of disease, ASD interventions, and sleep interventions. In addition, the internship director provided training on how to review an article and a template to guide the intern review process. Interns reported appreciating the opportunity to critically analyze articles individually and then discuss as a larger group with guidance from the internship director. The interns then co-wrote the first draft of the article review and the final draft was discussed and edited by the interns and the internship director. This extra time together between the internship director and interns allowed for frequent check-ins on training well-being outside of supervision and provided the internship director with some more intimate social exchanges with trainees.

As described in PWC-8 Supervision below, weekly inperson supervision and didactics were converted to a remote technology format. However, one barrier we encountered was supervisor availability to speak at pre-scheduled didactics due to competing demands related to COVID-19 (e.g., added clinical sessions to accommodate patients whose appointments had to be rescheduled due to cancelations in the early weeks of COVID-19). As a temporary solution, the internship director took on the vast majority of didactic lectures from May-July 2020 and documented these changes on the published didactics schedule. These didactics focused on evidence-based interventions for pediatric health issues and their psychosocial consequences (e.g., feeding disorders, physical abuse, sleep disorders, etc.). Each didactic included a thorough discussion of a research article relevant to each topic to ensure practice with critically evaluating research as well as an opportunity to restate what they learned in terms appropriate for sharing with patients and families. A drawback of this approach was some of the pre-scheduled topics had to be substituted for other topics to ensure content matter was within the purview of the internship director's clinical research expertise (e.g., a presentation on therapy with young adults with PTSD was replaced with content on pediatric feeding disorders). However, this approach allowed the program to meet many training elements related to the program's aims and the PWCs. Again, this adaptation allowed the internship director more frequent encounters with trainees, to check in on how they were coping with all the disruptions associated with COVID-19 as well as the escalation of social injustices related to police violence against Black lives.

Finally, the interns gained clinical research experience with the Helping Our Toddlers, Developing Our Children's Skills (HOT DOCS; Agazzi et al., 2020) parent-training program due to disruptions to their psychoeducational assessment rotation. More specifically, the interns co-facilitated virtual, small- and large-group HOT DOCS classes 6-8 h a week. This training opportunity temporarily replaced the assessment rotation, allowing interns to gain valuable experience in clinical research (e.g., informed consent, data collection, fidelity to model, telehealth adaptations), as well as clinical hours in interventions delivered via remote-based technology. This experience mitigated any loss of patient contact hours from the assessment rotation and resulted in an increase in client contact hours due to high attendance rates within the HOT DOCS program. Finally, this clinical research experience also provided an opportunity for interns to work with families from diverse communities (e.g., race, ethnicity, religion, family constellation, and socio-economic status). The interns reported great appreciation for this training opportunity as it allowed them to maintain the patient contact hours without worry and to practice their skills in teaching HOT DOCS with supervision from the internship director who is the principal investigator of HOT DOCS. Once again, this allowed for close monitoring of intern well-being during this difficult time. Supervisors were also grateful, as this opportunity alleviated supervisor feelings of worry and guilt that they would not identify adequate patient contact hours for inters during a very stressful time.

\section{PWC-2: Ethical and Legal Standards}

The COVID-19 pandemic introduced a host of ethical dilemmas and opportunities to apply ethical decision-making. Chenneville and Schwartz-Mette (2020) and Desai and Schwartz (2020) reviewed potential ethical issues and provided guidance to psychologists to conduct themselves in an ethical manner amidst the current pandemic and its aftermath. For example, these authors highlighted the relevance of Standard 3: Human Relations (Standard 3.01, Unfair Discrimination; American Psychological Association, 2017) in the APA ethics code, specifically the decisions psychologists face when providing equitable and fair clinical care (Chenneville \& Schwartz-Mette, 2020; Desai et al., 2020). In our training program, psychology supervisors and interns were deemed essential workers exempt from stay-at-home orders. Our internship director reached out to our trainee's academic institutions and shared our plans for adapting the training program and for keeping interns safe as essential workers. Our plan was supported by each trainees' director of clinical training at their academic institution. We were confronted with how to provide equitable and fair care for all patients including those who were at high risk due to their age, socioeconomic status, or employment. As COVID-19 cases were mounting, our practice plan swiftly implemented screening procedures to minimize the risk of transmission. Consistent with state guidelines from the Department of Health and licensing boards for medicine and psychology, our institution embraced COVID-19 screening procedures 
for all staff and patients, and PPE was required for everyone ages 2 and older. During face-to-face (F2F) interactions with patients, universal precautions were enforced such as physical distancing and the use of PPE and these precautions provided great comfort for internship staff and trainees. To ensure ethical decision-making related to trainee well-being, supervisors checked in with interns daily, as did the internship director. Trainees consistently reported comfort with their clinical roles given the extensive PPE and universal precautions, and reported appreciating the routine of patient services amidst the escalating uncertainty associated with the virus and the social injustices pervasive in the media. Over time and as cases swelled, patients and staff increasingly reported concerns with $\mathrm{F} 2 \mathrm{~F}$ care, and the transition to telehealth provided the greatest relief for all parties. Our practice plan had not embraced telehealth services prior to the pandemic, but as the Center for Medicaid and Medicare Services (CMS) expanded telehealth coverage it paved the way for our institution to embrace this technology. The initial transition to telehealth involved a steep learning curve as supervisors and interns alike learned new technology and discovered legal and ethical issues associated with telehealth and supervisory practices. Our Professional Integrity Office (PIO) granted permission for interns to continue providing clinical service to their patients via telehealth. At first, interns and supervisors were required to deliver the telehealth service in a private office in clinic. Over time, our PIO office became more familiar with state and federal laws around telehealth and eventually, our practice plan shifted all mental health providers to home-based telehealth, including our interns. This introduced ethical hurdles around supervision, which were addressed in multiple ways, as discussed below (see PWC-8: Supervision section).

The transition to telehealth also introduced new ethical and legal learning opportunities related to Standard 4: Privacy and Confidentiality. For example, Standard 4.01, Maintaining Confidentiality required psychologists and trainees working from home to identify a quiet and secure location wherein they could minimize confidentiality breeches. We worked with our trainees through free webinars from APA and articles on how to ensure client confidentiality by using headphones with a microphone and positioning themselves in a room away from others living in the home. Issues of consent and the limits of confidentiality (4.01, Discussing Limits of Confidentiality) were critical in relation to telehealth services. The potential for others to gain access to telehealth sessions or associated information was demonstrated by the Zoom privacy breaches wherein troublemakers hacked open Zoom meetings. Our practice plan embraced Microsoft Teams technology, due to its compliance with the Health Insurance Portability and Accountability Act (HIPPA). The migration to Teams was a reasonable learning curve for psychology supervisors and interns, who were quickly adept at supporting patients' ability to use the platform.

The shift to telehealth practices also required adaptation in our documentation and billing practices to conform with federal and state guidelines, as well as an understanding of the legal considerations around jurisdictional/interjurisdictional practice. Supervisors engaged interns in free webbased courses offered by the APA on these topics and the internship training director participated in daily virtual meetings with leaders of the university medical practice plan as they developed institutional policies on telehealth delivery of services, documentation and billing, and jurisdictional practice (e.g., our practice plan required patients to be at home at the address in the medical record for therapy services). The internship supervisors worked together to develop smart phrases for telehealth services (templates with required elements of a note) within the electronic medical record (EMR) to ensure that all notes included the levels of documentation required for billing. Interns were trained to use these smart phrases and their notes were scrutinized by supervisors to ensure compliance and accuracy with this new requirement. Supervisors paid special attention to the patient's location at time of service to ensure interns' services were complying with jurisdictional practice considerations, to ensure appropriate privacy during teletherapy (e.g., no teletherapy while shopping at Target or while on vacation in Georgia), and to ensure the patient could be located in the event of a safety emergency (e.g., at home address on file in medical record).

\section{PWC-3: Individual and Cultural Diversity}

At the onset of COVID-19, our interns had already participated in diversity didactics and had frequently discussed topics related to diversity through supervision. While this provided an opportunity to demonstrate a general understanding of their skills in respect to diversity, it had not provided an opportunity to apply their skills to a complex situation. As a result of COVID-19, various topics were coming up more frequently through the course of treatment such as: cultural beliefs related to medical decision-making, whether or not to engage in social distancing, whether or not to wear a mask and how to discuss COVID-19 with children. Supervisors and interns engaged in frequent conversations and supervision related to these topics. It was critical that supervisors were available to interns throughout the course of their day as often these conversations needed to take place outside of the typical scheduled supervision sessions. These opportunities allowed supervisors to model how to engage in new learning as many were learning how to have these conversations with patients right along with the interns.

As the COVID-19 pandemic continued there were also social injustices occurring across the US. This led to a 
greater intensity of thoughts and feelings for some patients related to these matters. Frequently, parents discussed news events with or in the presence of their children. Meanwhile, interns had to explore their own thoughts and feelings quickly and in detail in order to provide appropriate and sensitive treatment and recommendations to children and families. Supervisors provided additional time for interns to research current topics on cultural diversity. Supervision during this time took on a more reflective supervision model instead of a case consultation model in order for interns to process and discuss how they might put into practice what they had researched as well as discuss their own well-being. A document of resources for promoting conversations on race and racism was created and distributed to all psychologists and therapists within the practice. In addition, we explored in supervision how to support our patients with resource barriers and worked outside of supervision to compile local resources to share with patients needing information on food banks, shelter, utility assistance, shelter, and other public services. We circulated weekly a file published by our local Children's Board of Hillsborough County so that interns and faculty could share with patients in need.

\section{PWC-4: Professional Values, Attitudes, and Behaviors}

An unprecedented event, the COVID-19 pandemic resulted in an ever-changing terrain of uncertainty and the requirement of constant flexibility and adaptability. Desai et al. (2020) stressed the importance of remaining cognizant of the fact that psychology interns, who are concerned about their education and ability to meet training requirements while also adapting to treatment changes are not immune to the same stressors, anxieties, and uncertainties facing those for whom they are providing care. With these considerations in mind, supervisors implemented a stepwise approach to learning, which provided interns with educational opportunities to expand knowledge regarding service logistics (i.e., scheduling, billing, records, telehealth platforms), professional appearance and deportment, and behaviors needed to ensure continuity of care in accordance with evolving policies (i.e., shelter-in-place orders, essential workers, COVID curfew). Initial activities involved assigning interns webinars on telehealth best practices (Maheu, 2019, October 23) provided at no cost through the APA and other organizations. In addition, given the urgent need for family resources on coping with COVID-19 and social justice change as well as accessing mental health services during a crisis, interns were assigned self-study research and asked to create digital guides to be easily disseminated with patients.

One particular challenge experienced by interns involved engaging in self-reflection related to one's personal functioning. Scharff and colleagues (2020) found that trainees experienced difficulty navigating separation from their patients' concern about social distancing, loneliness related to isolation, and anxiety as well as helplessness about current events. Supervisors collaborated with interns to provide support, guidance, processing, and adaptive management of their own emotional distress and self-care. Indeed, selfcare and supervision are imperative to an intern's ability to engage in self-awareness and self-reflection, both of which are prerequisite skills for competency in professional values, attitudes, and behaviors (Desai et al., 2020; Scharff et al., 2020).

\section{PWC-5: Communication and Interpersonal Skills}

With the transition to a primarily telehealth modality, interns increased their awareness of nonverbal communication (i.e., facial expressions, body movements, head movements) and the implications of this for establishing and maintaining rapport as well as engaging the patient during session. Although there is evidence that therapeutic alliance quality, empathy, and participation in teletherapy may be comparable to inperson treatment (Irvine et al., 2020), interns faced challenges. For example, unreliable Wi-Fi or cellular service resulted in occasional interruptions. Also, interns found it difficult to maintain rapport, to engage patient and family during sessions, and to navigate the lack of control over the therapeutic environment. For example, patients often seemed extremely stressed during sessions. Frequently, patients requested to push appointment times, switched between tasks or walked away from the screen, refused to face the screen, or joined the session while driving or while in public places (e.g., the grocery store or Target). This required interns to refine their communication and interpersonal skills. Teletherapy during a pandemic altered the therapeutic alliance in ways both obvious and hard to quantify. This experience further increased interns' recognition of the importance of consultation, constructive feedback, and conflict resolution, all key elements of this PWC. Through supervision, interns were able to learn how to set clear boundaries, to approach treatment in a new creative way, and to manage their own emotional distress in order to empathize with and support their patients in the unfamiliar teletherapy world. Interns also developed greater autonomy and teletherapy-specific skills, self-reflection, and appreciation for their role as therapist.

\section{PWC-6: Assessment}

Throughout the training year, interns engage in numerous training elements to meet the assessment competency like discerning the need for assessment through a comprehensive diagnostic intake, selecting appropriate tools and adhering to administration standards, and utilizing assessment results 
to write integrated reports with intervention recommendations founded in evidence-based practices. The COVID-19 pandemic initially disrupted our program's ability to execute psychological assessment evaluations and thus, impacted interns' ability to refine assessment skills independently with supervision, as we were eight months into the internship year at the onset of the pandemic. Additionally, our institution's practice plan did not provide telehealth psychology services, which prevented supervisors from having knowledge and practice providing telehealth assessment as well as training prior to COVID-19. While the opportunity to provide this type of service and training during internship was ultimately positive, transitioning to telehealth was challenging. As Hewitt \& Loring (2020) noted, this type of transition requires programs to balance the demands of training with the demands of reviewing empirical literature for providing telehealth assessment administration, clinical service delivery, and maintaining the safety of patients, staff, and faculty.

With these changes, supervisors found themselves in a position where cultural factors, patient acceptance, technology barriers, and psychometric considerations regarding telehealth assessment intersected (Luxton et al., 2014). At the same time, this experience provided an opportunity for supervisors to collaborate with interns to demonstrate the application of the relevant research literature to clinical and psychometric decision-making and to implement plans specific to completion of diagnostic intakes, safety assessments (e.g., location at time of service, privacy for session, risk and safety planning), and psychological evaluations, which is consistent with the program's aims and the PWC related to assessment. Specifically, supervisors and interns found that assessment administration required a combination of F2F (with proper safety measures) and telehealth sessions. Given that some assessment measures (e.g., the Autism Diagnostic Observation Schedule, Second Edition) require the observation of facial expressions, our program discussed the possibility of having patients wear clear masks when telehealth administration was not possible. This circumstance allowed the training program to help foster and increase interns' psychological assessment abilities via telehealth, which previously was not an opportunity within the training program. During this training process, interns gained telehealth assessment knowledge by completing free web-based trainings provided by Pearson Q-Global and Riverside Insights before transitioning to remote-based technology administration. Throughout the remainder of the training year, interns and supervisors utilized supervision to discuss the potential limits of remote assessment and which portions of the evaluation battery could be executed within clinic, as the practice plan allowed for a small percentage of F2F service with appropriate safety and health precautions in place. Ultimately, the decision to conduct psychological assessments through remote locations allowed interns to continue participation in all rotations during the internship year and to expand the repertoire of their capabilities while adhering to best practices (Luxton et al., 2014).

\section{PWC 7: Intervention}

Prior to the COVID-19 pandemic, our interns participated in a variety of clinical and therapeutic settings, providing direct intervention services to patients and families. Specific intervention training included cognitive behavioral therapy (CBT), Parent-Child Interaction Therapy (PCIT), the HOT DOCS parent-training program, and individualized early intervention services for children under age 3 and their caregivers. However, the pandemic forced an immediate transition from F2F services with in vivo supervision to a telehealth treatment modality to protect the health and safety of healthcare providers and their patients. This transition introduced an obstacle to intern training in direct interventions, which caused some anxiety for supervisors and interns alike given there were only four months left in the training year. At the same time, it introduced an opportunity to add telehealth training elements to the curriculum to meet the intervention competency. As previously discussed, one curricular modification was the introduction of a series of selfguided webinars on telehealth services, as well as didactic instruction during supervision as we prepared interns for this new treatment platform.

As part of the early childhood assessment and intervention rotation, interns adjusted to delivering early intervention services via teletherapy. They were exposed to a variety of diverse home environments and family structures in which they were asked to model and coach interventions whose successful delivery largely depended upon the caregiver's ability to juggle a video call while being coached to work directly with their child with developmental delays. Interns learned and used parent coaching strategies to increase communication skills, such as modeling speech sounds, as well as ways to increase active engagement between parent and child in play, coaching parents to give effective directions, and responding appropriately to negative behaviors. The move from teaching and using these strategies in-person to telehealth taught interns how to use effective communication, ensuring that parents understood what they were directed to do, and efficiently providing helpful feedback for difficult therapeutic situations. Additionally, interns were able to observe more than actively playing or interacting with the child, which provided the parents with more opportunities to do and practice the skills, rather than simply listening and watching, then practicing later.

The pandemic situation further highlighted the need for interns to learn and use evidence-based interventions tailored to their patients' unique circumstances, specifically 
adapting interventions to better suit teletherapy. As there are instances in which making adaptations are appropriate, if not necessary (Malti et al., 2016), interns in this program received first-hand training to serve the needs of children and families from diverse backgrounds and socioeconomic situations. Via teletherapy, interns were tasked with learning greater flexibility and dealing with difficult client situations in a novel way.

The virtual therapy format forced trainees to learn how to provide effective therapy and interventions while also maintaining healthy boundaries with their patients. Video call technology that allowed for the application of 'blurred' or faux backgrounds helped interns maintain the distinction between a home and a 'home office' setting, especially for interns whose homes did not have a designated office space. Trainees had to learn how to read nonverbal cues via teletherapy. For instance, when a family participating in early intervention has not seen progress as expected, parents may disengage and reduce their involvement in the sessions, or even cancel or simply not show for telehealth sessions. When in person, it may be more obvious that the parent is unhappy; however, through a virtual meeting, interns have to be more aware of nonverbal overtures and respond accordingly, with often little context.

As interns progressed through their experiential training, they developed a stronger and more flexible version of what Geller (2020) refers to as 'therapeutic presence'- a way of being with the client, which provides greater opportunity for therapeutic growth. Although establishing a therapeutic presence may be challenging under normal circumstances, it can be even more difficult when the opportunity to be F2F with a client is restricted by the necessity either of wearing a mask or by conducting interventions remotely (Geller, 2020). However, it is still possible, and at times, even more necessary for the trainee therapist to focus on the patient and their needs. The flexibility required in approaching children and families from a therapeutic perspective with these necessary restrictions in place provided our interns with greater ability to establish rapport with patients and approach their individual needs sensitively and effectively.

Interns in our program also further developed their skills in tackling difficult topics and situations with sensitivity and appropriate timing, as patients have even more opportunity to leave telehealth sessions or avoid them altogether. Patients who are struggling to balance the myriad of responsibilities in their lives, such as working from home, e-learning, and caring for children who used to attend daycare or school will sometimes neglect teletherapy by avoiding phone calls and video sessions, or by canceling and refusing a rescheduled appointment. Trainees had to lean more on their supervisors to help guide them in their approach to these patients, and to work on meeting patients where they are able and willing to accept help. Sometimes, this required a reduction in services or spending some time during a session focusing on something other than the intended interventions. On the other hand, some patients struggled to maintain boundaries with their trainee therapists, reaching out to interns outside of normal treatment hours and seeking a more personal relationship. Establishing and maintaining professional boundaries, while also maintaining rapport and therapeutic presence with the client are practices that, although more challenging in the time of COVID-19, interns have immediate and consistent experience implementing while appropriately implementing therapeutic intervention strategies.

Part of our internship training program includes opportunities for interns to work with professionals from different disciplines, such as speech and language pathologists (SLPs) and occupational therapists (OTs), during evaluations, intervention sessions, and consultation sessions. Prior to COVID19, interns could observe as SLPs and OTs provided handson parent coaching, modeling how to form various speech sounds and demonstrate those for the child to copy, or how to provide sensory input for a child with Autism Spectrum Disorder. Wearing a mask innately prevents SLPs from being able to model the sounds and words for children and caregivers. Although telehealth removes the need to wear a mask, some children have a harder time focusing on a computer screen and engaging with the therapist, which drives greater caregiver involvement and a higher degree of coaching from the therapist. Interns are being exposed to more creative therapeutic techniques, such as the creation of social stories or a picture of a person making a sound, while the SLP or caregivers actually makes the sound for the child to see and hear. For our patients with developmental disabilities, such as ASD, interns gained a better understanding of how various factors can affect the level of communication, social engagement, and frustration tolerance a child may have, within the contexts of age, culture, socioeconomic status, and family dynamic.

\section{PWC-8: Supervision}

Prior to COVID-19, interns engaged in weekly in-person individual and group supervision and didactics. With the onset of COVID-19, a telesupervision policy was developed. The major adaptation involved converting supervision and didactics purely to a remote technology format for a temporary period. Materials for didactics lectures and supervision logs were uploaded to Box, a secure cloud content management service, for ease of sharing. Supervision forms were completed electronically and signed via DocuSign, which had the additional benefit of maintaining an electronic record of supervision logs and eliminating the need to scan paper files. As noted above, the transition to telehealth resulted in some ethical concerns. Some supervisors joined interns' telehealth sessions as a quiet observer for some part of the 
session, with verbal permission from the client and their caregiver which was documented in the EMR. Other supervisors switched to a co-therapy model wherein the supervisor and trainee worked side by side virtually and could debrief on issues at the conclusion of each session. Others provided real-time supervision by utilizing the private chat function during individual sessions. This surreptitious communication technique afforded by technology helped interns further develop their ability to incorporate feedback immediately, with little disruption to the flow of the therapy session. Regardless of supervision technique, cases were thoroughly discussed during individual and group telesupervision. As discussed by Bell et al. (2020), the expansion of telesupervision during the pandemic has offered great opportunities to enhance supervisory experiences, and the profession must consider it as a viable method even when no longer necessary.

\section{PWC 9: Consultation and Interprofessional/ Interdisciplinary Skills}

Given our program's location in a large academic medical center, we are able to provide a variety of training experiences in consultation and interprofessional collaborations. Our interns participate in major rotations in child and adolescent assessment and therapy, and a minor rotation in infant and early childhood assessment and intervention. Through these varied rotations, interns engage in interdisciplinary services alongside other providers (e.g., SLPs, OTs, case managers) as well as consult with a variety of medical professionals (e.g., physician residents, attending physicians, nursing staff) through clinical work, didactics, and grand rounds. These training elements result in collaborative learning across disciplines, and opportunities to hone effective communication skills. Prior to COVID-19, interns had frequent $\mathrm{F} 2 \mathrm{~F}$ contact with a variety of professionals, but the transition to remote-based work disrupted usual routines and usual interdisciplinary interactions. Although the move was necessary to protect the health and safety of all parties, the swiftness of the move left many unanswered questions. The lack of physical contact and ability to work in the same building with our colleagues affected trainees' opportunities to interact with each other and work directly with supervisors. The rapid shift raised anxiety as interns were uncertain about their schedules, how to support patients, and how COVID-19 may impact their graduation. Once telehealth practices were ready for launch, our program created opportunities for interns to engage in collaborative and interdisciplinary training and practices. For example, through shifting interns' clinical hours to provide group delivered parent training in the HOT DOCS program, we were able to pair interns with co-therapists from other disciplines, like early interventionists, and parent liaisons. This provided opportunities for our interns to hear the program content delivered from the perspective of another discipline. Interns are also able to participate in active collaboration and consultation between multiple disciplines within their early intervention rotation, through both eligibility evaluation clinics and intervention sessions. The shift to a more remote approach actually provides interns with more opportunities to see clinicians from other disciplines in action, observing how physical therapists (PTs) guide parents in helping a child with motor delays walk, as well as asking questions and offering suggestions when one on one with another clinician based on a child's needs. During evaluations, interns typically observe and participate in the administration of developmental assessments, as well as more disciplinespecific assessments for motor skills, communication, and difficult behavior. Although some of these other assessments are more limited through telehealth, interns are still experiencing working as part of a team with early interventionists, psychologists, SLPs, PTs, and OTs, which helps build their understanding of and relationships with other clinicians, expanding their comprehension of child development and pediatric therapies.

\section{Conclusion}

The trainee experience, with regard to intervention implementation and evaluation, has changed drastically since the onset of the COVID-19 pandemic and shows little sign of returning to its previous state any time soon. While it is impossible to predict a pandemic, COVID-19 has highlighted the importance of being prepared to adapt programs quickly and efficiently to ensure training across the nine PWCs. The adaptations we made allowed us to recognize the ability to increase access to care through telehealth for underserved populations and mentally and physically ill patients who found it difficult to leave their homes for inperson appointments in the past. Programs should continue to explore ways to have a flexible training model that can adapt to global pandemics or other emergencies. Our program's COVID-19 adaptations supported interns in their development of greater autonomy, self-confidence, and telepsychology skills, which is particularly important as each one transitions into their postdoctoral training year with the continuation of the COVID-19 pandemic.

Undoubtedly, like others in our field, our health psychology program learned many lessons during this pandemic. We close the article with a few recommendations for other programs to consider as our response to COVID19 evolves. First, look to professional associations, state licensing boards, and other agencies for guidance. As previously discussed, we greatly benefited from free webinars, policy statements, and updated guidance from these various 
agencies. Being aware of how others in health psychology were responding to different professional issues gave us solace that we were not alone, but also assurance that we were heading in the right direction. Second, keep discussions and learning about individual and cultural diversity central to all aspects of health psychology training. This pandemic affected all people differently and some groups were especially marginalized and at risk for physical and mental health complications associated with COVID-19. Training directors should consider ways to integrate diversity-informed training across rotations, activities, and experiences to better equip trainees to meet all patients' needs. Finally, embrace reflective practice in health psychology training. Our health service psychology training program's location in an urban academic medical center requires demanding schedules to serve complex patients, many of whom are from underserved communities. This work can be exhausting during usual times, but can quickly become overwhelming during a global pandemic when healthcare is as important as ever. Our supervisors had to rise to the [pandemic] occasion, like other healthcare professionals, and embracing reflective practices and reflective supervision with trainees provided a space to question our own ways of being, relating, and acting.

Author Contributions Each author in the byline agreed to serve as an author, approved the order of authorship presented herein, and accepted the responsibility of authorship including a substantial contribution to the manuscript.

Funding None.

Data availability None.

\section{Declarations}

Conflict of interest Authors Heather Agazzi, Shadae Najmabadi, Jacquelyn Flood, Danielle Cimorelli and Tiffany Chenneville declare that they have no conflict of interest.

Ethical approval None.

Informed consent None.

\section{References}

Agazzi, H., Childres, J., \& Armstrong, K. (2020). Helping our toddlers, developing our children's skills-4th edition (HOT DOCS). (4th ed.). University of South Florida, Department of Pediatrics, Section of Child Development
American Psychological Association. (2017). Ethical principles of psychologists and code of conduct. Retrieved from https://www. apa.org/ethics/code/

American Psychological Association. (2018). Program-specfic competencies: What they are and how to describe them. Commission on Accreditation Update. Retrieved from https://www.apa.org/ed/ accreditation/newsletter/2018/12/competencies

Association of Psychology and Postdoctoral and Internship Centers. (2020, March 23). COVID-19 Information [APPIC FAQs]. Retrieved from https://www.appic.org/News-Articles/ArtMID/ 1931/ArticleID/4/COVID-19-Information

Bell, D. J., Self, M. M., Davis, C., III., Conway, F., Washburn, J. J., \& Crepeau-Hobson, F. (2020). Health service psychology education and training in the time of COVID-19: Challenges and opportunities. American Psychologist, 75(7), 919-932

Chenneville, T., \& Schwartz-Mette, R. (2020). Ethical considerations for psychologists in the time of COVID-19. American Psychologist, 75(5), 644-654. https://doi.org/10.1037/amp0000661

Commission on Accreditation. (2017). Section C: IRs Related to the Standards of Accreditation. Retrieved from https://www.apa.org/ ed/accreditation/section-c-soa.pdf

Desai, A., Lankford, C., \& Schwartz, J. (2020). With crisis comes opportunity: Building ethical competencies in light of COVID19. Ethics \& Behavior, 30(6), 401-413. https://doi.org/10.1080/ 10508422.2020 .1762603

Geller, S. (2020). Cultivating online therapeutic presence: Strengthening therapeutic relationships in teletherapy sessions. Counselling Psychology Quarterly. https://doi.org/10.1080/09515070.2020. 1787348

Irvine, A., Drew, P., Bower, P., Brooks, H., Gellatly, J., Armitage, C. J., \& Bee, P. (2020). Are there interactional differences between telephone and face-to-face psychological therapy? A systematic review of comparative studies. Journal of Affective Disorders, 265, 120-131. https://doi.org/10.1016/j.jad.2020.01.057

Luxton, D. D., Pruitt, L. D., \& Osenbach, J. E. (2014). Best practices for remote psychological assessment via telehealth technologies. Professional Psychology: Research and Practice, 45, 27-35. https://doi.org/10.1037/a0034547

Maheu, M. (2019, October 23). Telepsychology Best Practice 101 [Webinar]. American Psychological Association. Retrieved from https://apa.content.online/catalog/product.xhtml?eid=15132

Malti, T., Noam, G. G., Beelmann, A., \& Sommer, S. (2016). Toward dynamic adaptation of psychological interventions for child and adolescent development and mental health. Journal of Clinical Child \& Adolescent Psychology, 45(6), 827-836. https://doi.org/ 10.1080/15374416.2016.1239539

Scharff, A., Breiner, C. E., Ueno, L. F., Underwood, S. B., Merritt, E. C., Welch, L. M., \& Litchford, G. B. (2020). Shifting a training clinic to teletherapy during the COVID-19 pandemic: a trainee perspective. Counselling Psychology Quarterly. https://doi.org/ 10.1080/09515070.2020.1786668

World Health Organization. (2020). Rolling updates on coronavirus disease (COVID-19). Retrieved from https://www.who.int/emerg encies/diseases/novel-coronavirus-2019/Events-as-they-happen

Publisher's Note Springer Nature remains neutral with regard to jurisdictional claims in published maps and institutional affiliations. 